\title{
Psychiatrische Diagnosesysteme, Konzepte psychischer Störungen, der zivilrechtliche Krankheitsbegriff und das Problem der neurokognitiven Störungen in ICD-11
}

\author{
Henning Saß ${ }^{1} \cdot$ Clemens Cording $^{2}$ \\ Eingegangen: 6. November 2020 / Angenommen: 11. November 2020 / Online publiziert: 11. Dezember 2020 \\ (c) Der/die Autor(en) 2020
}

\section{Zusammenfassung}

Die Revision von ICD-10 zu ICD-11 gibt Anlass, die Bedeutung der psychiatrischen Klassifikationen für rechtliche Fragestellungen zu überdenken. Kernpunkte sind dabei der Übergang vom grundlegenden Krankheitskonzept zu einem unverbindlichen Störungsbegriff (,disorder“) und die damit verbundenen Verunsicherungen der Beurteilungsnormen. Wenn die Zahl von Diagnoseschlüsseln, die für Zustände gestörten Befindens sowie psychischer und sozialer Dysfunktionen zur Verfügung stehen, immer mehr anwächst, stellt sich die Frage nach der forensischen Erheblichkeit. Kritisch zu sehen sind auch Tendenzen im Maßregelrecht, durch eine juristisch-funktionale Begriffsauslegung ,psychische Störung“ mit Gefährlichkeit gleichzusetzen. Dagegen wurde beim zivilrechtlichen Krankheitsbegriff eine Unabhängigkeit von den Änderungen diagnostischer Gepflogenheiten bewahrt.

Schlüsselwörter Klassifikationssysteme $\cdot$ Krankheitskonzepte $\cdot$ Psychische Störungen $\cdot$ Terminologie $\cdot$ Forensische Psychiatrie

\section{Psychiatric diagnosis systems, concepts of mental disorders, the civil law disease concept and the problem of neurocognitive disorders in ICD-11}

\begin{abstract}
The revision of the International Classification of Diseases (ICD) from ICD-10 to ICD-11 gives occasion to reconsider the importance of the psychiatric classifications for legal questions. The core points are the transition from the fundamental disease concept to a noncommittal disorder term and the associated uncertainty of the assessment standards. When the number of diagnosis keys, which are available for emotional disturbances as well as mental and social dysfunctions, continues to increase, the question of the forensic relevance arises. Tendencies in the right of criminal commitment by a juristic functional interpretation of terms to equate "mental disorder" with dangerousness, must also be critically viewed. In contrast, an independence from the modifications of diagnostic conventions was preserved by the disease concept in civil law.
\end{abstract}

Keywords Classification systems $\cdot$ Disease concepts $\cdot$ Mental disorders $\cdot$ Terminology $\cdot$ Forensic psychiatry

Prof. Dr. med. Henning $\mathrm{Sa}$

hsass@ukaachen.de

1 Klinik für Psychiatrie, Psychotherapie und Psychosomatik, Universitätsklinikum der RWTH Aachen, 52074 Aachen,

Deutschland

2 Psychiatrische Universitätsklinik, Universitätsstr. 84, 93053 Regensburg, Deutschland 


\section{Vorbemerkung}

Der stete Wandel der psychiatrischen Klassifikationen ist auf der einen Seite wissensgetrieben, beruht aber auf der anderen Seite auch auf sozialen Prozessen, die sowohl innerhalb der beteiligten Wissenschaften wie in der gesamten Gesellschaft wirksam sind. Er hat Einfluss auf den $\mathrm{Zu}$ gang zu Leistungen des Gesundheitssystems, auf die Gestaltung von klinischen Abläufen und die Finanzierung von Forschungsvorhaben, schließlich auch auf den forensischen Dialog zwischen Rechtswissenschaften und den begutachtenden Disziplinen. Grundsätzlich zu beachten ist der Unterschied zwischen rechtlichen Normierungen und diagnostischen Gepflogenheiten. Im Zivilrecht mit dem Fokus auf der freien Willensbestimmung haben sich die Prüfungsverfahren und Beurteilungskriterien mittlerweile sehr stark von den diagnostischen Systemen mit all ihren Ausfächerungen und dem Vordringen in leichter ausgeprägte Störungsbereiche gelöst. Im Strafrecht mit dem Fokus auf der Einsichtsund Steuerungsfähigkeit kommt es dagegen leichter zu einer Verunsicherung, weil die Orientierung an den psychiatrischen Erkrankungen im engeren Sinne aufgegeben und ein unspezifischer Störungsbegriff zum Vehikel für funktionale Normsetzungen durch rechtliche Instanzen wurde. All dies ist im Folgenden vor dem Hintergrund der bevorstehenden Einführung von ICD-11 zu erörtern, wobei ein Schwerpunkt auf die Krankheits- und Störungsbegriffe gelegt wird.

\section{Zur Problematik wechselnder Krankheitskonzepte}

Bei den forensisch-psychiatrischen Begutachtungen spielt die Frage einer evtl. vorliegenden Krankheit bzw. Störung eine wichtige Rolle als Eingangsvoraussetzung der ersten Ebene des stets zweistufigen Beurteilungsverfahrens. Einen einheitlich definierten, allgemeingültigen Krankheitsbegriff gibt es allerdings weder in der Medizin noch im deutschen Recht (Häfner 2019; Beck 2017, S. V). Für unterschiedliche Anwendungen und Zwecke existieren unterschiedliche Krankheitsbegriffe nebeneinander, und diese ändern sich im Zeitverlauf infolge neuer wissenschaftlicher Erkenntnisse, aber auch gesellschaftlicher Entwicklungen. Alle Krankheitsbegriffe haben empirische und normative Wurzeln in unterschiedlichem Verhältnis, die rechtlichen sind stärker normativ (,Soll“) angelegt, die medizinischen stärker auf die vorfindbare Realität („Ist“) ausgerichtet (Roxin 1986; Wieland 2004). Alle bilden einerseits gewisse Aspekte der Realität ab, dienen aber auch bestimmten Zwecken und schaffen damit begriffliche Entitäten, die wiederum in die Realität hineinwirken.
In der forensischen Psychiatrie war lange Zeit die wegen ihrer Klarheit gerade bei Juristen einflussreiche Krankheitslehre von Kurt Schneider die wesentliche Orientierungsgröße. Er favorisierte einen empirischen Dualismus, ohne zu einer metaphysischen Auslegung des Leib-Seele-Verhältnisses Stellung zu nehmen, wenn er formulierte, Krankheit gebe es nur im Leiblichen, und „krankhaft" sei seelisch Abnormes dann zu nennen, wenn es auf krankhafte Organprozesse zurückzuführen sei (K. Schneider 1967). Auch die Zyklothymie und Schizophrenie ordnete er, obwohl die verursachenden Krankheitsvorgänge nicht bekannt waren, den krankhaften Störungen zu, da es ein sehr gut gestütztes Postulat und eine sehr gut begründete Hypothese sei, dass ihnen Krankheiten zugrunde liegen. Von den seelischen Abnormitäten als Folge von Krankheiten unterschied er abnorme Spielarten seelischen Wesens, zu denen nach heutiger Diktion etwa neurotische und Persönlichkeitsstörungen oder auch Paraphilien zu rechnen wären.

Diesem strikt medizinischen und somatopathologisch begründeten Krankheitsbegriff begegnete in der Reformdebatte im Vorfeld der Strafrechtsreform von 1975 zunehmend eine Kritik, die schon damals zu Divergenzen zwischen medizinischem, juristischem und sozial orientiertem Krankheitsbegriff führte (Lange 1963). So wurde in der forensischen Psychologie die Orientierung am Modell der normalen Handlung bzw. einer ungestörten Entwicklung der sozial-kulturellen Persönlichkeit gefordert (Undeutsch 1974), während Rasch (1982) die Einbeziehung der soziologischen und kriminologischen Dimension in einen „sozial-strukturellen Krankheitsbegriff“ vornahm.

Bekanntlich führten derartige Strömungen zu einer Ausweitung des Krankheitsverständnisses mit dem juristischen Krankheitsbegriff in der Strafrechtsreform von 1975. Psychische Krankheiten (im engeren Sinne K. Schneiders) fielen nun in die Kategorie "krankhafte seelische Störung“ der Schuldfähigkeitsparagraphen, während für die im Sinne K. Schneiders nicht krankhaften, gleichwohl aber vom juristischen Krankheitsbegriff umfassten Zustände die beiden neuen Kategorien der ,tiefgreifenden Bewusstseinsstörung“ und der „schweren anderen seelischen Abartigkeit“ entstanden.

\section{Aktuelle Krankheits- und Störungsbegriffe}

In der gegenwärtigen medizintheoretischen Diskussion werden sowohl psychische als auch physische Krankheiten definiert durch objektive Krankheitszeichen, subjektives Leiden, Funktionsbeeinträchtigung und Störung der sozialen Teilhabe, dies allerdings nicht unbedingt gleichzeitig und nicht immer in einem Krankheitsverlauf (Boorse 1976; Schramme 2012). Als Konsequenz für die psychiatrische Klassifikation ergibt sich daraus bei Heinz (2014, S. 118, 
348), dass als psychische Krankheiten im engeren Sinne nur die endogenen und exogenen Psychosen (inklusive Demenzen) sowie die schweren, substanzgebundenen Suchtkrankheiten gelten, denn nur davon werden die vorgenannten Kriterien regelhaft erfüllt. Dagegen werden die anderen Bereiche psychischer Störungen in einer überraschenden Übereinstimmung mit der Systematik von K. Schneider als Variationen menschlichen Erlebens bezeichnet. Letztere weisen fließende Übergänge von allgemeinen menschlichen Eigenschaften und Reaktionsweisen zu diagnostizierbaren psychischen Störungen auf. Beispiele dafür umfassen Neurosen und Persönlichkeitsstörungen, ferner posttraumatische Belastungsstörungen, soziale Phobien, Störungen der Impulskontrolle und leichtere Verstimmungen.

Eine neue Facette in den zwischen Juristen und Medizinern divergierenden Auffassungen zum Krankheitsbegriff entstand mit den Diskussionen um die ,psychische Störung" im $\S 1$ des seit dem 01.01.2011 gültigen Therapieunterbringungsgesetzes (ThUG; Kröber 2011; Saß 2011; Koller 2019). In einer von der Rechtspolitik gesehenen Notlage, die durch Urteile des Europäischen Gerichtshofs für Menschenrechte zur Frage der Sicherungsverwahrung und der ,true mental disorder“ entstanden war, wurde in diesem Gesetz das Konstrukt einer ,psychischen Störung“" geschaffen, die Eingangsvoraussetzung für eine Unterbringung gefährlicher Personen sein sollte. Dieser juristisch legitimierte Begriff der „,psychischen Störung“ decke, so hieß es in der Gesetzesbegründung, ein breites Spektrum von psychischen Erscheinungsformen ab, von denen nur ein Teil in der forensisch-psychiatrischen Begutachtungspraxis als psychische Erkrankung gewertet werde (BT-DRS 17/3403, 52f.). Zur Erläuterung hieß es dort weiter, auch ein abnorm aggressives und ernsthaft unverantwortliches Verhalten könne eine psychische Störung im Sinne des ThuG darstellen. In ähnlicher Richtung formulierte das Bundesverfassungsgericht zu dieser speziellen „psychischen Störung“, es handle sich dabei ungeachtet der Bezugnahme auf ICD-10 und DSM-IV um einen unbestimmten Rechtsbegriff, der mit den überkommenen Kategorisierungen in der Psychiatrie nicht deckungsgleich sei. Ob die Merkmale im Einzelnen erfüllt seien, hätten die Gerichte eigenständig zu prüfen (BVerfG, Beschluss vom 13.07.2013 - 2 BvR 2302/11 u.a.).

In den Kontext der divergierenden Auffassungen zum Krankheits- bzw. Störungsbegriff, die vor Kurzem auch in dieser Zeitschrift dargelegt wurden (Borchard und Gerth 2020; Habermeyer et al. 2020), gehören ferner die gegenwärtigen Diskussionen in der Schweiz über die Definition einer „schweren psychischen Störung“, die seit 2007 eine Eingangsvoraussetzung für therapeutische Unterbringungsmaßnahmen im Schweizer Rechtssystem darstellt. Die Dynamik dieser Debatten wird weniger von theoretisch-konzeptionellen Überlegungen als vielmehr von der praktischen Zielsetzung bestimmt, eine langfristige Sicherung von ge- fährlichen Straftätern zu gewährleisten (Habermeyer et al. 2019). Auch in den dortigen Auseinandersetzungen werden die unterschiedlichen Positionen zu Krankheits- bzw. Störungsbegriffen in Psychologie und Psychiatrie zum Anlass genommen, eine eigene, rechtlich ausgerichtete Definition der „schweren psychischen Störung“ vorzunehmen, wobei von juristischer Seite betont wird, dass der Begriff funktionaler Natur sei, da er sich nach dem Zweck der Maßnahme richte (Schweizer Bundesgericht, Beschluss vom 03.10.2019 - 6B_933/2018).

Hier dient also in vergleichbarer Weise, wie es in Deutschland bei der Schaffung des ThUG geschehen ist, eine neue, rechtspolitisch orientierte Definition des Begriffes „psychische Störung“ dazu, die Verfolgung von kriminalpolitisch opportun erscheinenden Zielen zu ermöglichen. Dies ist, ähnlich wie es bei den Debatten um die Strafrechtsreform von 1975 sowie den Auseinandersetzungen um die Sicherungsverwahrung und Therapieunterbringung der Fall war, ein weiteres ein Beispiel für die naturgemäBen Friktionen zwischen juristischem und medizinischem Krankheits- bzw. Störungsverständnis, in Abhängigkeit weniger von wissenschaftlichen Erkenntnissen, sondern vielmehr von normativen Zwecken und gesellschaftlichem Zeitgeist.

$\mathrm{Zu}$ den Verwerfungen zwischen medizinischen und juristischen Krankheitskonzeptionen, die in den skizzierten Entwicklungen deutlich werden, hat die Psychiatrie durch die Begriffswahl in ihren Klassifikationssystemen insofern beigetragen, als dort die nosologischen Grundsatzfragen eher pragmatisch-defensiv behandelt wurden und keineswegs so didaktisch-prononciert, wie es bei K. Schneider der Fall gewesen war. So wurde in der gesamten ICD-10Klassifikation der etwas unglückliche Begriff der ,psychischen Störung“ (,,mental disorder") verwendet, um, wie es hieß, den problematischen Gebrauch von „Krankheit“ oder „Erkrankung“ weitgehend zu vermeiden. Zur ,psychischen Störung" wurde festgestellt, dies sei kein exakter Begriff, vielmehr handle es sich um einen klinisch erkennbaren Komplex von Symptomen oder Verhaltensauffälligkeiten, die immer auf der individuellen und oft auch auf der Gruppen- und sozialen Ebene mit Belastungen und Beeinträchtigungen von Funktionen verbunden sind. Für die forensische Anwendung von Bedeutung war die Feststellung, dass soziale Abweichungen oder soziale Konflikte allein nicht als psychische Störung im Sinne von ICD-10 angesehen werden sollten.

Auch im DSM-System wurde weitgehend, obschon nicht ganz konsequent auf den Krankheitsbegriff verzichtet und stattdessen ebenfalls von „psychischer Störung“ gesprochen. Diese wird im aktuellen DSM-5 definiert als ein Syndrom, das durch klinisch bedeutsame Störungen in den Kognitionen, der Emotionsregulation oder des Verhaltens charakterisiert wird, wobei diese Störungen Ausdruck von 
dysfunktionalen psychologischen, biologischen oder entwicklungsbezogenen Prozessen sind, die den psychischen und seelischen Funktionen zugrunde liegen (APA 2013). Weiteres definierendes Merkmal ist, dass psychische Störungen typischerweise verbunden sind mit bedeutsamem Leiden oder Behinderung hinsichtlich sozialer und berufs-/ ausbildungsbezogener sowie anderer wichtiger Aktivitäten. Ähnlich wie in ICD-10 gibt es rechtlich relevante Einschränkungen, etwa wenn es heißt, sozial abweichendes Verhalten (z.B. politischer, religiöser und sexueller Art) und Konflikte zwischen Individuen und Gesellschaft seien keine psychischen Störungen, sofern nicht eine der oben genannten Dysfunktionen zugrunde liege, auch sage eine Diagnose nichts aus über das Ausmaß der verminderten Verhaltenskontrolle.

In der neuen ICD-11 der WHO finden sich analoge Formulierungen zur Definition der psychischen Störungen, die dort im Kapitel 06 als „,mental, behavioural and neurodevelopmental disorders" aufgeführt sind (https://icd.who.int/ en). Diese stellen Syndrome dar, die durch klinisch signifikante Beeinträchtigungen eines Individuums in den Bereichen der Kognition, der Emotionsregulation und des Verhaltens charakterisiert sind, wobei dies auf eine Dysfunktion in psychologischen, biologischen oder Entwicklungsprozessen zurückgeht, die den psychischen Funktionen und der Verhaltenssteuerung zugrunde liegen. Auch hier heißt es, dass die Störungen üblicherweise mit Leiden oder einer Funktionsbeeinträchtigung hinsichtlich persönlicher, familiärer, sozialer, ausbildungs- und berufsbezogener oder anderer wichtiger Funktionsbereiche einhergehen.

Dieser kurze Überblick soll zeigen, dass die verschiedenen Definitionen von Krankheit bzw. psychischer Störung ebenso wenig wie die diagnostischen Konventionen in den jeweiligen Klassifikationssystemen eine sichere Basis für die Beantwortung der forensisch-psychiatrischen Fragestellungen darstellen. Das gilt umso mehr, wenn man die ständigen Veränderungen durch die regelmäßigen Revisionen im ICD- und im DSM-System berücksichtigt. Deshalb sind sowohl im strafrechtlichen wie im zivilrechtlichen Begutachtungsbereich je eigenständige Systematiken für die Überprüfung der Schuldfähigkeitsfrage oder der freien Willensbestimmung entstanden. Wichtiger als die sich wandelnden diagnostischen Gepflogenheiten sind dabei die zentralen, in beiden Bereichen gültigen Grundsätze der zweistufigen Methode im Sinne K. Schneiders (1948) und eines psychopathologischen Bezugssystems für den Vergleich der Erheblichkeit der festgestellten Störungen (vergl. Saß 1991, 2008). Daneben gibt es etwas andere Begutachtungsregeln für die Einwilligungsfähigkeit (Snellgrove und Steinert 2017); auch hat sich nach dem Urteil des Bundesverfassungsgerichts zur Suizidhilfe mit dem Konzept der Freiverantwortlichkeit ein weiterer eigenständiger Bereich konturiert (Cording und $\mathrm{Sa}$ 2020).

\section{Zur Bedeutung der Diagnosesysteme für die forensische Psychiatrie}

Die neueren psychiatrischen Klassifikationen haben zwar zu einer Verbesserung der Kommunikation innerhalb der Psychiatrie geführt und erleichtern sicherlich auch die Verständigung mit Nachbardisziplinen einschließlich der Justiz, doch darf es nicht zu einem kritiklos-dogmatischen Umgang mit den je aktuellen diagnostischen Instrumenten kommen (Saß 1990; Hoff 1992). Theoretisch bedeutsam ist die Gefahr einer „Reifizierung“ oder „Ontologisierung“ seelischer Störungen, bei der der Konstruktcharakter der operational definierten diagnostischen Einheiten verwechselt wird mit gleichsam natürlich vorgegebenen, unveränderbaren, „real existierenden“ Krankheitseinheiten. Ferner wurde von juristischer Seite früh bemerkt (Blau 1989), dass die starke Erweiterung des Kataloges seelischer Störungen in den neueren Klassifikationssystemen zu einer „Psychiatrisierung des Alltags" führt, ähnlich wie später von einem der Protagonisten des DSM-Systems und Hauptherausgeber des DSM-IV eine „Inflation psychiatrischer Diagnosen“ beklagt wurde (Frances 2013). Forensisch ist daran wesentlich, dass durch die gewachsene Zahl codierungsfähiger Störungen und die damit verbundenen Probleme der sog. Komorbidität Missverständnisse begünstigt werden, bei denen aus der Zahl der Diagnosen fälschlich auf Schwere und Erheblichkeit der festgestellten Auffälligkeiten geschlossen wird.

Deshalb ist gerade aus forensisch-psychiatrischer Sicht auch weiterhin vor einer Überschätzung von Wandlungen in den diagnostischen Konzeptionen zu warnen. Psychiatrische Klassifikationssysteme bilden keine letztgültigen Wahrheiten über psychische Störungen ab, sondern dienen lediglich als zeitgebundene Konventionen der Verständigung über abnorme psychische Phänomene ( $\mathrm{Saß} 2015)$. Ihre Kriterien und Kategorien sind stets auf bestimmte praktische Zwecke hin optimiert und können daher allenfalls unter Nützlichkeitsaspekten bewertet werden. Die ICD-Klassifikation hatte zu Beginn ihren Hauptnutzen in der nationalen und internationalen Gesundheitsberichterstattung sowie in der Epidemiologie, während sie erst in jüngerer Zeit mehr auf klinisch-therapeutische Belange ausgerichtet ist. Bei dem DSM-III und seinen Revisionen lag das Schwergewicht auf dem Nutzen für die wissenschaftlichen Fragestellungen. Für beide Klassifikationssysteme gilt, dass sie nicht im Hinblick auf rechtliche Fragestellungen konzipiert wurden.

Wegen der in einzelnen Staaten sehr unterschiedlichen Rechtssysteme und auch wegen der Verschiedenheiten der Rechtsgebiete Straf-, Zivil- und Sozialrecht untereinander können internationale Diagnosesysteme niemals auf die jeweils relevanten Merkmale für die forensischen Begutachtungen hin optimiert werden. Entsprechende Warnhinweise 
für die Verwendung im rechtlichen Kontext sind in allen Auflagen von DSM-III bis DSM-5 ausdrücklich formuliert. In ICD-10 war dies bislang nicht so klar ausgesprochen, doch sind keine Gründe ersichtlich, warum die Argumente aus dem DSM-System nicht auch uneingeschränkt für ICD10/11 gültig sein sollten.

\section{Zu den Neuerungen in ICD-11}

Die Weltgesundheitsorganisation (WHO) hat im Juni 2018 eine Revision der „International Classification of Diseases and Related Health Problems" (ICD-11) verabschiedet, die im Mai 2019 von der 72. Weltgesundheitskonferenz angenommen wurde mit der Perspektive, dass sie zum Januar 2022 von den 194 Mitgliedsstaaten eingeführt werden soll (WHO 2018). Allerdings liegen bislang nur die klinischen Beschreibungen in der endgültigen Form vor, nicht aber operationale Kriterien für die Diagnosen und Schweregrade. Auch eine offizielle deutsche Übersetzung steht noch nicht zur Verfügung, sodass die in diesem Text verwandten Termini als vorläufig anzusehen sind.

Der umfangreiche, etwa 10-jährige Revisionsprozess für das psychiatrische Kapitel der ICD-11 beruhte auf einer breiten internationalen Beteiligung, Multilingualität, Multidisziplinarität und Partizipation (Reed et al. 2019). Hauptprinzipien waren dabei die klinische Brauchbarkeit und eine globale Anwendbarkeit. Dabei wurde durch personelle Überschneidungen in den Arbeitsgruppen für die Vorbereitung sowohl von ICD-11 als auch von DSM-5 eine Harmonisierung zwischen diesen beiden Systemen angestrebt. Dennoch wird es auch in Zukunft bei zwei konkurrierenden Systemen für die Diagnostik von psychischen Störungen bleiben, wobei DSM-5 die Forschungsliteratur dominiert, während ICD-10 bzw. künftig ICD-11 weiterhin in vielen Staaten das offiziell gültige System für Dokumentation und Statistik im Gesundheitswesen darstellt.

Die psychischen, verhaltensbezogenen und neurokognitiven Störungen in ICD-11 enthalten 21 Störungsgruppen, während es in ICD-10 lediglich 11 Störungsgruppen waren. Wichtig erscheint, dass ICD-11 wie bereits DSM-5 die Differenzierung der psychischen Störungen nach Lebensspannen aufgibt, sodass es keine Unterscheidung mehr zwischen einem Kapitel für Erkrankungen im Kindes- und Jugendalter bzw. im Erwachsenenalter gibt. Ferner sind in ICD-11 die kulturbezogenen Informationen deutlich stärker berücksichtigt als in der Vorgängerversion. Ähnlich wie DSM-5 führt auch ICD-11 an verschiedenen Stellen dimensionale Ansätze zur Ergänzung des grundsätzlich weiterhin kategorialen Systems ein. Die Begründung lautet, dass die meisten psychischen Störungen sich besser durch miteinander interagierende symptomatologische Dimensionen beschreiben lassen als durch diskrete Kategorien (Krueger et al.
2018; Reed et al. 2019). Einen revolutionären Umsturz bedeutet dies allerdings nicht, da der grundsätzlich kategoriale Ansatz beibehalten wird und die Klassifizierung der psychischen Phänomene weiterhin auf den Angaben der Patienten über ihre Beschwerden oder auf klinisch beobachtbaren Symptomen beruht (Gaebel et al. 2020).

$\mathrm{Zu}$ den gerade für die Forensik wichtigen Veränderungen in ICD-11 gehört die lange umstrittene Neustrukturierung im Kapitel der Persönlichkeitsstörungen (Tyrer et al. 2015; Reed 2018; Jeung-Maarse und Herpertz 2020; Habermeyer und Herpertz in diesem Heft). Problematisch erscheint aus forensischer Sicht die später noch zu diskutierende Neukonzipierung der organischen psychischen Störungen im Kapitel der „,neurokognitiven Störungen“. Neu aufgenommen wurden einige potenziell auch forensisch relevante Störungen, etwa ,gaming disorder“, ,,compulsive sexual behavior disorder" und ,intermittent explosive disorder".

Mit der Ausweitung des Kataloges der psychischen Störungen liegt ICD-11 auf einer ähnlichen Linie wie DSM-5 und birgt sicherlich die Gefahr, dass es zu einer generellen Erhöhung der registrierten Prävalenz von psychischen Störungen in der Gesamtbevölkerung kommt. Immerhin aber soll eine möglichst exakte Fassung der wesentlichen klinischen Merkmale in den ,klinischen Beschreibungen und diagnostischen Leitlinien“ (,clinical descriptions and diagnostic guidelines“) dazu dienen, eine Abgrenzung zwischen zu diagnostizierenden Störungen und bloßen Variationen menschlicher Funktionsweisen $\mathrm{zu}$ ermöglichen. Hierauf wird bei der forensischen Anwendung sowohl im straf- wie im zivilrechtlichen Bereich besonders zu achten sein.

\section{Zivilrechtlicher Krankheitsbegriff und ICD-11}

Psychiatrische Begutachtungen zu Fragestellungen des Zivilrechts betreffen v.a. die Geschäftsunfähigkeit und ihre Unterformen ( $\S 104,105,827,1304,1314,2229$ BGB) sowie die Betreuungs- und die Unterbringungsbedürftigkeit ( $\S 1896$ ff., 1906 BGB). Anders als im Strafrecht geht es hier nicht um den Schutz Dritter, sondern um den sog. Erwachsenenschutz und die Fähigkeit der Betroffenen zur Selbstbestimmung (Lipp 2017). Für die zivilrechtlichen Begutachtungsfragen gilt im Wesentlichen derselbe rechtliche Krankheitsbegriff (Lipp 2017); einzelne Modifikationen im Betreuungsrecht und im Hinblick auf Freiverantwortlichkeit bei assistiertem Suizid sind im vorliegenden thematischen Zusammenhang nicht wesentlich.

Aufgabe des psychiatrischen Sachverständigen als $B e$ rater des Gerichts (BGH NJW 1998, 3355 f.; Lipp 2017) 
im Zivilprozess und auch im FamFG ${ }^{1}$-Verfahren ist es, dem Gericht den Expertenkonsens der forensischen Psychiatrie in Bezug auf die Gutachtensfrage, angewandt auf den konkret zu begutachtenden Einzelfall, zu vermitteln. Juristen und Psychiater haben einen unterschiedlichen Erfahrungshintergrund und unterschiedliche Denktraditionen, sie sprechen gleichsam verschiedene Sprachen - selbst dann, wenn sie, wie oben diskutiert, gelegentlich dieselben Termini verwenden. Um sich dem Gericht verständlich machen zu können, müssen forensisch tätige Psychiater sich in beiden Denkgebäuden und Begrifflichkeiten hinreichend auskennen: Sie sollen als Dolmetscher zwischen den Disziplinen fungieren (Cording und Nedopil 2014, S. 6). Die Definitionsmacht über die Fragestellung und die anzuwendenden grundsätzlichen Beurteilungskriterien liegt dabei stets aufseiten der Justiz.

Die medizinischen Diagnosenklassifikationssysteme ändern sich in kürzeren Abständen und dann häufig abrupter als die sich eher langsam weiterentwickelnde Rechtsprechung. Anders als im deutschen Strafrecht mit seinen Reformen von 1965 bis 1975 hat sich an dem seit 120 Jahren bestehenden zivilrechtlichen Krankheitsbegriff bisher kaum etwas geändert, auch wenn die Rechtsprechung der letzten 30 Jahre hinsichtlich der Beurteilungskriterien einige Präzisierungen und Ausdifferenzierungen vorgenommen hat.

Zum zivilrechtlichen Krankheitsbegriff heißt es im aktuellen Kommentar von Staudinger und Klumpp zur Geschäftsfähigkeit:

$\S 104$ Nr. 2 setzt einen normativen Krankheitsbegriff. Dieser ist nicht ... auf organisch bedingte Krankheitsbilder begrenzt, sondern an das Vorliegen einer geistigen Anomalie gekoppelt .... Wann diese Anomalie erworben wurde oder ob sie angeboren ist, spielt ebenso wenig eine Rolle ... wie ihre Ursachen - maßgeblich ist eine Abnormität und damit ein Abweichen vom als normal Angesehenen. Deshalb findet der Krankheitsbegriff des $§ 104$ Nr. 2 seine Grenzen auch nicht in den vorgegebenen Krankheitsbildern der medizinischen Kategorisierung und erst recht nicht in den dort verwendeten Krankheitstermini ... - der normative Krankheitsbegriff ist gerade kein naturwissenschaftlicher. Die medizinische Forschung gibt für die Festlegung einer krankhaften Störung aber selbstredend wichtige Hinweise, um die Abweichung und damit die Anomalie zu erkennen und damit den juristischen Krankheitswert einer Störung festzulegen .... Damit ist die krankhafte Störung letztlich der empirisch feststellbare Ausgangspunkt für die Geschäftsunfähigkeit. ... Die anormale geistige Störung ist final

\footnotetext{
${ }^{1}$ Gesetz über das Verfahren in Familiensachen und in den Angelegenheiten der freiwilligen Gerichtsbarkeit
}

festzustellen, die sie auslösenden Ursachen sind irrelevant. (Staudinger und Klumpp 2017, § 104 BGB, Rn 10 und 11; Hervorhebungen hinzugefügt)

In der vorangegangenen Auflage war erläutert worden, was mit final gemeint ist:

Entscheidend für die Krankhaftigkeit einer psychischen Störung im Sinne des für § 104 Nr. 2 maßgeblichen juristischen Krankheitsbegriffs ist ... der Grad, das Ausmaß der Störung im Hinblick auf die Möglichkeit eines Ausschlusses der freien Willensbestimmung ...; es kommt darauf an, ob die betreffende Anomalie hinsichtlich ihrer Wirkungen denjenigen psychischen Störungen gleichwertig ist, an deren krankhaften Charakter innerhalb der zuständigen Erfahrungswissenschaften kein Zweifel besteht .... Diese Gleichwertigkeit kann mit dem Begriff des "Krankheitswertes“ umschrieben werden. (Staudinger und Knothe 2012, $\S 104$ BGB, Rn 8; Hervorhebungen hinzugefügt)

Dass die im Zivilrecht immer noch üblichen Bezeichnungen „geistige“ Anomalie oder „Geistesstörung“ keine Einengung auf intellektuelle bzw. kognitive Störungen bedeutet, sondern gerade und v.a. auch Störungen voluntativer und affektiver Funktionen einschließen, ist ständige Rechtsprechung (z.B. BGH NJW 1953, 1342; BGH NJW 1970, $1680 \mathrm{f}$.). Derartige gesetzliche Formulierungen beruhen auf dem jeweiligen Stand der Medizin, die der Gesetzgeber vorfand, als er diese Regelungen schuf (Lipp 2017); in diesem Fall gehen sie auf die 1890er-Jahre zurück.

Der Krankheitsbegriff der $\S \S 104,105,2229$ BGB ist nicht an bestimmte Ursachen gekoppelt, sondern an psychopathologische Funktionsstörungen, die nach Art und Intensität geeignet sind, eine freie Willensbestimmung im Sinne der zivilrechtlichen Rechtsprechung auszuschließen (Details bei Cording 2014, S. $37 \mathrm{f}$; Cording und Roth 2015). Dadurch besteht eine gewisse Ähnlichkeit mit den psychiatrischen Klassifikationssystemen ICD-10, DSM-IV und DSM-5, deren deskriptiv-operationale Diagnosekriterien sich ebenfalls vorwiegend auf die klinisch feststellbaren psychopathologischen Symptome stützen, und nur ausnahmsweise auf die (organische) Ätiologie (Zabel 2017). Auch besteht hier eine deutliche Parallele zu dem Vorschlag des psychopathologischen Referenzsystems ( $\mathrm{Sa}$ 1991, 2008). Die Ähnlichkeit der Ansätze sowie die mit inzwischen etwa 25 Jahren ungewöhnlich lange Geltungsdauer der ICD-10 haben dazu beigetragen, dass diese medizinische Klassifikation im Gegensatz zu den früheren ICD-Versionen und auch zum DSM-System von der Rechtsprechung als Möglichkeit einer ersten Orientierung bei der gutachtlichen Prüfung der ersten, nosologischen Beurteilungsebene anerkannt wurde und vielfach in konkrete Gerichtsentscheidungen eingegangen ist (z.B. OLG 
München ZEV 2017, 148; Staudinger und Knothe 2012, Rn 7; Staudinger und Klumpp 2017, Rn 10). Diese obergerichtlichen Entscheidungen werden durch den Wechsel zu ICD-11 natürlich nicht obsolet. Das alles ändert freilich nichts daran, dass letztlich nicht der medizinische oder ICD-basierte Krankheitsbegriff für die Beurteilung zivilrechtlicher Fragestellungen maßgeblich ist, sondern allein der rechtliche. Diagnosen nach psychiatrischen Klassifikationssystemen wie ICD oder DSM können dem Gutachter im Einzelfall helfen, sind aber weder notwendig noch hinreichend für die Beantwortung dieser Gutachtensfragen (Cording und $\mathrm{Sa} \beta$ 2017).

\section{Zum Problem der neurokognitiven Störungen}

Für viele der bei Begutachtungen im Zivilrecht wichtigen Störungskategorien - Schizophrenien, bipolare Störungen, unipolare Depressionen, (protrahierte) Delirien - bringt ICD-11 wohl keine gravierenden Änderungen. Schwierigkeiten bereitet allerdings der Wegfall wichtiger Krankheitskategorien im Bereich neurokognitiver Störungen, die gerade für die zivilrechtliche Begutachtung eine erhebliche Relevanz haben. Dass die ätiologisch, klinisch und in ihren Verlaufscharakteristika höchst heterogenen vaskulären Demenzen (Wetterling 1998; Schmidtke 2006, S. $208 \mathrm{ff}$.) in der ICD-11 nur noch als einheitliche Kategorie (6D81 Dementia due to cerebrovascular disease) ohne weitere Unterteilung aufgeführt werden, ist nicht nachvollziehbar. Vaskuläre Demenzen sind die zweithäufigste Demenzform, und von diesen ist die chronisch-progressive, mikroangiopathisch bedingte subkortikale vaskuläre Demenz (ICD-10 F01.2, auch Binswanger Enzephalopathie) die weitaus häufigste Form (Roh und Lee 2014; Wetterling 2016, S. 58); sie ist zugleich die wichtigste nichtdegenerative Demenzform (Schmidtke 2006, S. 211). Unter den zur Begutachtung der Testierfähigkeit kommenden Probanden sind die Fälle subkortikaler vaskulärer Demenzen bzw. subkortikal-kortikaler Mischformen nach eigener Erfahrung sogar deutlich häufiger als reine Alzheimer-Demenzen (Cording 2014, S. 61). Psychopathologisch unterscheidet sich diese Demenzform zudem von den Alzheimer-Demenzen, sodass die daran ausgerichteten, allgemein üblichen Demenzkriterien hier nur eingeschränkt zutreffen und auch Standardtests wie der Mini-Mental-State-Test (MMST) deutlich weniger Aussagekraft haben (Schmidtke 2006, S. 213 f., 218 f.; Cording 2014, S. $60 \mathrm{ff} ., 102$ ).

Leider ist in der ICD-11 auch eine Kategorie für die organisch bedingten Wesens- bzw. Persönlichkeitsänderungen (etwa nach schweren Schädel-Hirn-Traumen oder chronischem Alkoholismus), also die organischen Persönlichkeitsstörungen gemäß ICD-10 F07.0 bzw. F10.71, nicht mehr vorgesehen, obwohl diese klinisch und forensisch eine erhebliche Rolle spielen (z.B. Cording 2014). Dasselbe gilt für die (allerdings eher seltenen) endoformen organischen Psychosen gemäß ICD-10 F06.0-F06.3. Insgesamt erscheint die Differenzierung der organisch bedingten Störungen in der ICD-11 gegenüber den Kategorien F01, F06 und F07 der ICD-10 unangemessen reduziert.

Wenn man als Sachverständiger in den genannten Fällen nach ICD-11 keine adäquaten Diagnosen stellen kann, wird man auf die Kategorien und Kriterien der wissenschaftlichen Literatur, der fachlichen Leitlinien und/oder der ICD10 zurückgreifen müssen, um von dort aus das Vorliegen der Voraussetzungen für den zivilrechtlichen Krankheitsbegriff (erste Beurteilungsebene) zu prüfen.

Die Schwierigkeiten der Begutachtung von den (im Zivilrecht sehr seltenen) Fällen extremer Zuspitzungen etwa bei Borderline-Persönlichkeitsstörungen (Herpertz et al. 1996; Cording 2014, S. 92) betreffen dagegen nicht die erste, diagnostische Beurteilungsebene, sondern die zweite Ebene der jeweils vorliegenden psychopathologischen Funktionsbeeinträchtigungen - diese Schwierigkeiten hängen nicht vom verwendeten Diagnoseklassifikationssystem $\mathrm{ab}$ und werden sich mit der ICD-11 nicht ändern.

Es wurde kritisiert, dass ICD-11 bei der AlzheimerErkrankung weder im Kapitel 06 (Psychiatrie) noch im Kapitel 08 (Neurologie) die neue biomarkerbasierte Klassifikation integriert hat, sondern sich weiterhin nach klinischsyndromalen Kategorien ausrichtet (Jessen und Frölich 2018). Dies eröffnet einen interessanten Blick auf die Zukunft, die für die organischen psychischen Störungen eine Renaissance ätiologisch definierter Krankheitsentitäten auf nunmehr molekularer Basis erwarten lässt. Das wird für die frühzeitige Anwendung damit einhergehender Therapieoptionen wichtig sein, es aber auch notwendig machen, sorgfältiger als bisher zwischen zugrunde liegendem Morbus (z.B. Alzheimer-Krankheit) und aktueller klinisch-phänotypischer Erscheinungsform und Funktionsbeeinträchtigung (z. B. Demenz) zu unterscheiden. Für den Krankheitsbegriff des Zivilrechts wird sich dadurch nichts ändern, denn für die gutachterliche Einschätzung der freien Willensbestimmung ist nach wie vor die forensischpsychopathologische Analyse der mentalen Funktionen entscheidend, und nicht die biologischen Ursachen und die medizinische Krankheitsdiagnose (Cording 2014; Saß 2015).

\section{Fazit}

Nachdem die in den verschiedenen Rechtsgebieten durchaus unterschiedlichen rechtlichen Krankheitsbegriffe nicht an die jeweils gültigen Klassifikationssysteme gebunden sind, ändert sich durch die Einführung der ICD-11 (ebenso 
wie schon bisher bei DSM-5) aufseiten der rechtlich vorgegebenen Rahmenbedingungen nichts. Ebenso wenig ändert sich selbstverständlich faktisch an den zur Beurteilung kommenden Fällen und deren psychopathologischen Funktionsstörungen. Was sich mit der verbindlichen Einführung von ICD-11 ändern wird, sind allein die medizinischen Diagnosekategorien und die dafür geltenden Kriterien. Das betrifft die psychiatrische Routinediagnostik und die Gesundheitsberichterstattung. Die forensisch-psychiatrische Begutachtung der straf- und zivilrechtlichen Fragestellungen ist davon nicht im Kern betroffen, weil sich weder die (lediglich mit neuer medizinischer Terminologie bezeichneten) Sachverhalte ändern, noch deren Beurteilung in rechtlicher Hinsicht. Allerdings wird in der Übergangsphase die „Übersetzungsleistung" von den neuen klinischen in die gewohnten rechtlichen Kategorien erst neu erlernt werden müssen.

Funding Open Access funding enabled and organized by Projekt DEAL.

Interessenkonflikt H. Saß und C. Cording geben an, dass kein Interessenkonflikt besteht.

Open Access Dieser Artikel wird unter der Creative Commons Namensnennung 4.0 International Lizenz veröffentlicht, welche die Nutzung, Vervielfältigung, Bearbeitung, Verbreitung und Wiedergabe in jeglichem Medium und Format erlaubt, sofern Sie den/die ursprünglichen Autor(en) und die Quelle ordnungsgemäß nennen, einen Link zur Creative Commons Lizenz beifügen und angeben, ob Änderungen vorgenommen wurden.

Die in diesem Artikel enthaltenen Bilder und sonstiges Drittmaterial unterliegen ebenfalls der genannten Creative Commons Lizenz, sofern sich aus der Abbildungslegende nichts anderes ergibt. Sofern das betreffende Material nicht unter der genannten Creative Commons Lizenz steht und die betreffende Handlung nicht nach gesetzlichen Vorschriften erlaubt ist, ist für die oben aufgeführten Weiterverwendungen des Materials die Einwilligung des jeweiligen Rechteinhabers einzuholen.

Weitere Details zur Lizenz entnehmen Sie bitte der Lizenzinformation auf http://creativecommons.org/licenses/by/4.0/deed.de.

\section{Literatur}

APA (American Psychiatric Association) (2013) Diagnostic and statistical manual of mental disorders (DSM-5). American Psychiatric Publishing, Arlington (Deutsche Ausgabe: Falkai P, Wittchen H-U, Doepfner M, Gaebel W, Maier W, Rief W, Saß H, Zaudig M (Hrsg.) (2015) Diagnostisches und Statistisches Manual Psychischer Störungen DSM-5. Göttingen, Hogrefe)

Beck S (Hrsg) (2017) Krankheit \& Recht. Ethische und juristische Perspektiven. Springer, Berlin

Blau G (1989) Methodologische Probleme bei der Handhabung der Schuldfähigkeitsbestimmungen des Strafgesetzbuches aus juristischer Sicht. Monatsschr Kriminol Strafrechtsreform 72:71-77

Boorse C (1976) What a theory of mental health should be. J Theory Soc Behav 6:61-84

Borchard B, Gerth J (2020) Die Relevanz eines kohärenten forensischen Beurteilungs- und Behandlungsprozesses. Forens Psychiatr Psychol Kriminol 14:197-211

Cording C (2014) Geschäftsfähigkeit und ihre Unterformen. In: Cording C, Nedopil N (Hrsg) Psychiatrische Begutachtungen im Zi- vilrecht - Ein Handbuch für die Praxis. Pabst Science Publishers, Lengerich, S 29-128

Cording C, Nedopil N (Hrsg) (2014) Psychiatrische Begutachtungen im Zivilrecht - Ein Handbuch für die Praxis. Pabst Science Publishers, Lengerich

Cording C, Roth G (2015) Zivilrechtliche Verantwortlichkeit und Neurobiologie - ein Widerspruch? Neue Jur Wochenschr 68:26-31

Cording C, Saß H (2017) Standards und Fehler bei der Begutachtung der Geschäfts- und Testierfähigkeit. Forens Psychiatr Psychol Kriminol 11:228-233

Cording C, Saß H (2020) Die Freiverantwortlichkeit der Entscheidung für einen assistierten Suizid. Neue Jur Wochenschr 73:2695-2697

Frances A (2013) Saving normal: an insider's report against out-ofcontrol psychiatric diagnosis, DSM-5, big pharma, and the medicalization of ordinary life. William Morrow, New York (Deutsch 20154: Normal. Gegen die Inflation psychiatrischer Diagnosen, Dumont, Köln)

Gaebel W, Stricker J, Kerst A (2020) Changes from ICD-10 to ICD-11 and future directions in psychiatric classification. Dialogues Clin Neurosci 22(1):7-13

Habermeyer E, Lau S, Hachtel H, Graf M (2019) Der Begriff der schweren psychischen Störung: Eine alternativlose Höhenmarke. In: Heer M, Habermeyer E, Bernard S (Hrsg) Die schwere psychische Störung als Voraussetzung von therapeutischen Maßnahmen. Stämpfli, Bern, S 43-57

Habermeyer E, Mokros A, Briken P (2020) „Die Relevanz eines kohärenten forensischen Beurteilungs- und Behandlungsprozesses“: grosser Wurf oder alter Wein in undichtem Schlauch? Forens Psychiatr Psychol Kriminol 14:212-219

Häfner H (2019) Psychische Krankheit - ein Mehrregionenbegriff. Fortschr Neurol Psychiatr 87:685-694

Heinz A (2014) Der Begriff der psychischen Krankheit. Suhrkamp, Berlin

Herpertz S, Erkwoh R, Saß H (1996) Die Suizidproblematik aus psychiatrisch-psychotherapeutischer Sicht. In: Pohlmeier $\mathrm{H}$, Schöch H, Venzlaff U (Hrsg) Suizid zwischen Medizin und Recht. G. Fischer, Stuttgart Jena New York, S 1-12

Hoff P (1992) Neuere psychiatrische Klassifikationssysteme und ihre Bedeutung für die forensische Psychiatrie. Gesundheitswesen 54:244-250

Jessen F, Frölich L (2018) ICD-11: Neurokognitive Störungen. Fortschr Neurol Psychiatr 86:171-177

Jeung-Maarse H, Herpertz S (2020) Neues zur Diagnostik und Therapie von Persönlichkeitsstörungen - Änderungen in ICD-11. Nervenarzt 91:863-871

Koller M (2019) Der Begriff der schweren psychischen Störung in der deutschen und europäischen Rechtsprechung. In: Heer M, Habermeyer E, Bernard S (Hrsg) Die schwere psychische Störung als Voraussetzung von therapeutischen Maßnahmen. Stämpfli, Bern, S 99-135

Kröber H-L (2011) „Psychische Störung“ als Begründung für staatliche Eingriffe in Grundrechte des Individuums. Forens Psychiatr Psychol Kriminol 5:234-243

Krueger R, Kotov R, Watson D et al (2018) Progress in achieving quantitative classification of psychopathology. World Psychiatry $17: 282-293$

Lange R (1963) Der juristische Krankheitsbegriff. In: Bürger-Prinz G, Giese H (Hrsg) Beiträge zur Sexualforschung. Heft 28: Die Zurechnungsfähigkeit bei Sittlichkeitsstraftätern. Enke, Stuttgart, S $1-20$

Lipp V (2017) Krankheit und Autonomie im Zivilrecht. In: Beck S (Hrsg) Krankheit \& Recht. Springer, Berlin, S 171-196

Rasch W (1982) Angst vor der Abartigkeit. Neue Jur Wochenschr 2:177-224

Reed G (2018) Progress in developing a classification of personality disorders for ICD-11. World J Psychiatry 17:227-229

Reed G, First M, Kogan C, Hyman S, Gureye O et al (2019) Innovations and changes in the ICD-11 classification of mental, be- 
havioural and neurodevelopmental disorders. World Psychiatry 18(1):3-19

Roh JH, Lee JH (2014) Recent updates on subcortical ischemic vascular dementia. J Stroke 16:18-26

Roxin C (1986) Schuldunfähigkeit Erwachsener im Urteil des Strafrechts. In: Eisenmenger W et al (Hrsg) Medizin und Recht. Festschrift für Wolfgang Spann. Springer, Berlin

Saß H (1990) Operationalisierte Diagnostik in der Psychiatrie. Nervenarzt 61:255-258

$\mathrm{Saß} \mathrm{H}$ (1991) Forensische Erheblichkeit seelischer Störungen im psychopathologischen Referenzsystem. In: Schütz H, Kaatsch H-J, Thomsen H (Hrsg) Medizinrecht-Psychopathologie-Rechtsmedizin. Springer, Berlin, Heidelberg, S 266-281

$\mathrm{Saß} \mathrm{H}$ (2008) Psychische Störungen und Schuldfähigkeit - Ein psychopathologisches Referenzsystem. Psychiatrie 5(3):182-189

Saß H (2011) „Psychische Störung“ zwischen Medizin und Recht: Anything goes? Forens Psychiatr Psychol Kriminol 5:129-131

$\mathrm{Sa} ß \mathrm{H}$ (2015) Psychiatrische Klassifikationssysteme und Forensische Psychiatrie. Forens Psychiatr Psychol Kriminol 9:127-129

Schmidtke K (2006) Demenzen. Reihe Klinische Neurologie. Kohlhammer, Stuttgart

Schneider K (1948) Die Beurteilung der Zurechnungsfähigkeit. Thieme, Stuttgart

Schneider K (1967) Klinische Psychopathologie, 8. Aufl. Thieme, Stuttgart

Schramme T (2012) Die Begriffe „Gesundheit“ und „Krankheit“ in der philosophischen Tradition. In: Schramme T (Hrsg) Krankheitstheorien. Suhrkamp, Berlin, S 9-37
Snellgrove BJ, Steinert T (2017) Einwilligungsfähigkeit vor dem Hintergrund der UN-Behindertenrechtskonvention. Forens Psychiatr Psychol Kriminol 11:234-243

Staudinger JV, Klumpp S (2017) Kommentar zum BGB. De Gruyter, Berlin

Staudinger JV, Knothe H-G (2012) Kommentar zum BGB. De Gruyter, Berlin

Tyrer P, Reed G, Crawford M (2015) Classification, prevalence and effect of personality disorders. Lancet 385:717-726

Undeutsch U (1974) Schuldfähigkeit unter psychologischem Aspekt. In: Eisen G (Hrsg) Handwörterbuch der Rechtsmedizin. Enke, Stuttgart, S 91-115

Wetterling T (1998) Vaskuläre Demenz - ein schlüssiges Konzept? Z Gerontol Geriat 31:36-45

Wetterling T (2016) Freier Wille und neuropsychiatrische Erkrankungen. Kohlhammer, Stuttgart

WHO (World Health Organization) (2018) International statistical classification of diseases and related health problems (11 th revision). https://icd.who.int/en. Zugegriffen: 1. Nov. 2020

Wieland W (2004) Grundlegende Aspekte des Krankheitsbegriffs. In: Mazous N, Werner MH, Wiesing U (Hrsg) Krankheitsbegriff und Mittelverteilung. Nomos, Baden-Baden, S 15-29

Zabel B (2017) Der Krankheitsbegriff des § 20 StGB. Entwicklungen im Spannungsfeld von Psychiatrie, Philosophie und Strafrecht. In: Beck S (Hrsg) Krankheit \& Recht. Springer, Berlin, S 135-168 